\title{
Enseñar Sociología a quienes no estudian Sociología
}

\author{
Sandra Obiol-Francés ${ }^{a}$, José Beltrán Llavador ${ }^{b}$ Zira Box Varela ${ }^{c}$, Daniel Gabaldón \\ Estevand, Francesc J. Hernàndez Dobon e, Ignacio Martínez Morales ${ }^{\mathrm{f}}$, Juan Ramón \\ Martínez Morales. \\ ${ }^{a}$ Dept. Sociologia i Antropologia Social, Universitat de València. Sandra.Obiol@uv.es \\ ${ }^{\text {b }}$ Dept. Sociologia i Antropologia Social, Universitat de València Jose.Beltran@uv.es \\ ${ }^{\mathrm{c}}$ Dept. Sociologia i Antropologia Social, Universitat de València. Zira.Box@uv.es \\ ${ }^{\mathrm{d}}$ Dept. Sociologia i Antropologia Social, Universitat de València Daniel.Gabaldon@uv.es \\ ${ }^{\mathrm{e}}$ Dept. Sociologia i Antropologia Social, Universitat de València. Francesc.J.Hernandez@uv.es \\ ${ }^{\mathrm{f}}$ Dept. Sociologia i Antropologia Social, Universitat de València. Ignacio.Martinez@uv.es \\ g Dept. Sociologia i Antropologia Social, Universitat de València. Juan.R.Martinez@uv.es
}

\begin{abstract}
La imaginación sociológica (Wright Mills, 1993) es fundamental en el ejercicio de la docencia. No se pueden entender las acciones individuales de los diferentes agentes de la comunidad educativa sin conocer su posición en la estructura social. El objetivo de esta propuesta es que los estudiantes del Grado de Maestra/o de Educación Infantil (Universitat de València) logren esta mirada estructural. El proyecto parte de la metodología de clase inversa y lo integra en un proceso de contraste del conocimiento teórico con la práctica diaria de los y las maestras a partir de la elaboración y análisis de entrevistas.
\end{abstract}

\section{Keywords:}

Imaginación sociológica, metodologías activas, estructura social, clase inversa, Educación Infantil

\footnotetext{
Abstract

The sociological imagination (Wright Mills, 1993) is fundamental in the teaching profession. It is not possible to understand individuals' actions of the different education agents without knowing their place in the social structure. The objective of this proposal is that the students of the Degree in Preschool Education (University of Valencia) achieve this point of view. The project starts with a flipped classroom methodology in combination with other active methodologies to end contrasting scientific knowledge with the daily teacher's work, through the realization and analyses of interviews.
}

\section{Keywords:}

Sociological imagination, active methodologies, social structure, flipped classroom, Preschool Education. 


\section{Introducción}

La imaginación sociológica para Wright Mills (1993) -autor que acuñó el concepto en 1959es la capacidad de analizar, de comprender, la biografía personal incluida en una sociedad y en una sucesión histórica; esa es, nos dice, "su tarea y su promesa" (Wright Mills, 1993, p.26). Se trata de que las personas puedan captar a través de la imaginación sociológica "lo que está ocurriendo en el mundo y comprender lo que está pasando en ellos mismos como puntos diminutos de las intersecciones de la biografía y de la historia dentro de la sociedad" (Wright Mills, 1993, p.27). Se trata de tomar conciencia de que nuestra manera de pensar y actuar individualmente está condicionada por la posición que ocupamos en la sociedad y nos une a todas aquellas personas con las que compartimos esta misma posición social. O dicho de otro modo, que la posición que nosotros ocupamos en el mundo influye decisivamente en nuestras acciones individuales, incluso en la construcción de nuestra identidad.

Evidenciar la importancia de esta capacidad es el principal propósito que se infiere del diseño y contenido de la asignatura Estructura Social y Educación del primer curso del grado de Maestro/a de Educación Infantil y Primaria en la Universidad de València en su Facultad de Magisterio. Se trata, a nuestro entender, de una habilidad imprescindible para la tarea docente pues procura una mayor comprensión de los niños y niñas, de las familias, de los gestores administrativos y políticos, y también de uno mismo como maestra/o. No en vano, la adquisición de esta capacidad de relación entre individuo y sociedad debería ser fundamental en cualquier curso introductorio de Sociología. En consecuencia, el objetivo del curso fue, claramente, guiar al estudiantado en el proceso de descubrimiento de que la estructura social da forma a los comportamientos humanos y a sus experiencias. Precisamente la estructura social es un constructo teórico para poder captar la complejidad de las relaciones que se establecen entre las diferentes posiciones sociales ordenadas jerárquicamente. Es un instrumento teórico que nos ha de servir para entender mejor cómo las personas de manera individual y colectiva accedemos de manera diferenciada - desigual- a los recursos existentes en nuestra sociedad. Y también cómo las personas formamos parte activa de estas relaciones de desigualdad, como bien señala el mismo Wright Mills (1993, p. 25): "Hemos llegado a saber que todo individuo vive, de una generación a otra en una sociedad, que vive una biografía y que la vive dentro de una sucesión histórica. Por el hecho de vivir contribuye, aunque sea en pequeñísima medida, a formar a esa sociedad y al curso de su historia, aun cuando él está formado por la sociedad y por su impulso histórico."

El trabajo con los estudiantes de este concepto pretende que tomen conciencia, crítica, del papel de la escuela -y con ésta, del docente- en la reproducción de la desigualdad social. De hecho, la relación de la escuela con las diferentes desigualdades sociales ha sido ampliamente refrendada a lo largo de los años en el ámbito de la Sociología de la Educación (Beltrán y Hernández Dobón, 2011; Fernández Palomares, 2009). La escuela no es neutra en términos de desigualdad, no en vano es un agente fundamental en los procesos de socialización (Ariño y Serra, 2010). Especialmente si tenemos en cuenta que estamos hablando de la educación infantil y primaria, aquellos niveles donde la función instructiva de la escuela, aun siendo importante, no lo es tanto como la transmisión de valores y prácticas culturales de la comunidad de referencia. 
El objetivo principal de la asignatura es poner en evidencia la relación de la escuela con las variables básicas de esta desigualdad: clase social, género y etnia fundamentalmente. Considerar la imaginación sociológica como una competencia básica en la formación de futuros maestros y maestras contribuye a formar educadores reflexivos y críticos acerca de su misma práctica profesional y de la relación con otros agentes de la comunidad educativa. Se trata de un reto tanto en el ámbito de la enseñanza universitaria como en su prolongación en su práctica profesional. Este artículo presenta una propuesta de renovación pedagógica en este sentido. A partir de la combinación de trabajo autónomo de los estudiantes y de la elaboración de pequeñas investigaciones de base sociológica he intentado que los estudiantes de la asignatura de Estructura Social y Educación adquieran una actitud crítica ante el proceso educativo. Como bien apuntan Hironimus-Wendt y Wallace (2009), la imaginación sociológica de Wright Mills es un concepto teórico con clara referencia su aplicabilidad práctica, requiere poder ser llevado a la vida real de los estudiantes. Por ese motivo la propuesta metodológica que presento requiere el contraste de los conceptos teóricos con la realidad cotidiana de los maestros/as con el fin último que adquieran imaginación sociológica.

La comunicación se estructura en tres grandes apartados. En primer lugar, se exponen las principales consideraciones pedagógicas en las que se ha sustentado la propuesta de innovación pedagógica: el aprendizaje autónomo y colaborativo. En segundo lugar, se plantea la propuesta de renovación metodológica llevada a cabo. Para terminar, como conclusión, se analizan los resultados obtenidos en este planteamiento y los retos de futuro.

\section{Objetivos: fomentar la autonomía en el aprendizaje}

Cada inicio de curso el objetivo principal de los profesores es conseguir la atención y compromiso de sus estudiantes. Es indudable que no existe una receta milagrosa para ello. Además, se ha de tener en cuenta que cada curso nos enfrentamos a estudiantes cada vez más jóvenes y, por tanto, más alejados de las experiencias generacionales del docente. Por ese motivo no son pocos los autores que defienden un cambio en las metodologías docentes que consigan una implicación mayor de los estudiantes en el proceso de aprendizaje complementando o descartando metodologías más tradicionales que basculan en la relación profesor-emisor activo de conocimiento/estudiante-receptor pasivo de ese mismo conocimiento (Forsey et al. 2013; Bain, 2006; Reidsema, Hadgraft i Kavanagh, 2017).

Este artículo se sitúa precisamente ante la necesidad de repensar -que no abandonar- las clases magistrales más tradicionales animados por la dificultad que tiene el hecho de impartir una materia que, a nuestro entender, es imprescindible para los maestros de infantil y primaria pero que no se encuentra entre los intereses mayoritarios e iniciales de los mismos, por tratarse de una disciplina complementaria a lo que podríamos considerar el núcleo duro de su formación. Y la propuesta resultante de esta reflexión es una transformación de la manera de organizar la docencia basada en la combinación de la clase magistral, la clase inversa (flipped classroom) y la necesidad de contraponer, desde una perspectiva crítica, los conocimientos teóricos a la práctica profesional de la docencia. Una propuesta metodológica compleja surgida a partir de la conjunción de elementos que consideramos que pueden 
resultar de utilidad para captar mejor el interés del estudiantado con el que compartimos unos pocos meses cada curso.

La propuesta supone la disolución de la frontera espacial que marcan las paredes del aula en la adquisición de conocimiento teórico. Precisamente el concepto de clase invertida se basa en esta disolución. Supone, según Lage, Platt y Teglia (2000), autores a quienes se les atribuye la creación del concepto, en que aquello que se ha hecho tradicionalmente fuera del aula entre al aula y aquello que se ha realizado tradicionalmente dentro del aula salga de la misma. Siempre con la ayuda que proporciona el uso de la tecnología, un aspecto importante cuando nos referimos a estudiantes jóvenes para quienes sus relaciones cotidianas con el mundo que les rodea pasan en gran medida por las nuevas tecnologías y las redes sociales.

El funcionamiento básico de la clase invertida es sencillo: se trata de que los estudiantes trabajen fuera del aula materiales en los que se encontrarán los conocimientos teóricos básicos de la materia. Una vez hecho este trabajo, y ya dentro del aula, las posibles dudas serán tratadas con la guía del profesor a través de una exposición más tradicional o bien a través de otras actividades formativas de carácter más práctico. Actividades que permitan un aprendizaje colaborativo, como se expondrá en la propuesta, que ha demostrado sus resultados positivos con la combinación de otras metodologías (Angelini, 2016). Es decir, en el aula se utiliza el tiempo con otros propósitos que el meramente expositivo y permite optimizar el uso del tiempo siendo tarea del profesor consolidar aquello previamente aprendido fuera del aula.

El trabajo del profesor en el aula por tanto será la consolidación del conocimiento, no la presentación del mismo, con el valor añadido que se puede hablar en el mismo idioma que los estudiantes, puesto que ya se habrán familiarizado con la terminología propia de la materia así como con las ideas básicas de los temas elegidos (Bergmann y Sams, 2012). Con esto se modifica el papel tradicional del docente, asumiendo en mayor medida el rol de facilitador, de guía en el proceso de aprendizaje de los estudiantes que adquieren un mayor protagonismo en este proceso (Bergmann y Sams, 2012). Además, se trata de una metodología, según Lage, Platt y Teglia (2000) que favorece adaptarse a una gran parte de los diferentes perfiles de aprendizaje que pueden existir en un aula, combinando diferentes metodologías como las clases magistrales tradicionales, el trabajo autónomo o bien -como es el caso que se propone- el trabajo colaborativo. En definitiva, se trata de que los estudiantes asuman la responsabilidad de su aprendizaje (Bain, 2006; Reidsema, Hadgraft y Kavanagh, 2017).

La Sociología, como apuntan Forsey et al. (2013) no ha sido precisamente conocida por encabezar el paso de metodologías tradicionales a este tipo de metodologías más complejas. Sin embargo, podemos encontrar un número importante de iniciativas que precisamente tratan de poner en contacto el conocimiento teórico con la vida cotidiana (Hironimus-Wendt, Wallace, 2009; Hsiung, 2008; Huisman, 2010; Greenfield, 2006; Hattery, 2003). En este sentido, proponemos a nuestros estudiantes que construyan un camino propio de descubrimiento del conocimiento que procure una consolidación mayor de éste que el que se consigue con el uso, únicamente, de metodologías tradicionales. Se trata de un compromiso 
imprescindible con una asignatura que trata de evidenciar las condiciones sociales en las que se ejerce el magisterio.

\section{Acercarse a la escuela como método de aprendizaje}

\subsection{DESCRIPCIÓN DE LA PROPUESTA}

La propuesta de renovación metodológica que aquí se presenta se ha llevado a cabo durante tres cursos (2016-17, 2017-18 y 2018-19) y cuenta desde el curso 2017-18 con el reconocimiento de la Universitat de València como proyecto de innovación educativa (UVSFPIE_RMD17-588310 IMAGINACIÓ SOCIOLÒGICA I ESCOLA). Se basa en la asignatura de Estructura Social y Educación en el Grado de Maestra/o de Educación Infantil en la Universitat de València que forma parte de las materias básicas de la titulación, en su primer curso, y cuenta con un peso curricular de seis créditos. Según la memoria de verificación de la titulación, los contenidos de la materia en la que se basa la propuesta de renovación metodológica suponen un repaso a las principales variables y procesos que dan forma a las estructuras sociales contemporáneas en la sociedad occidental. Además, entre sus competencias específicas encontramos la necesidad de comprender las bases de la relación de los diferentes agentes de la comunidad educativa, en especial de las familias. En este sentido se propone una metodología de aprendizaje activo y centrada en el estudiante basada en el método de clase inversa. La propuesta se desarrolla en cinco fases que fueron plasmadas en un cronograma, minuciosamente planeado y presentado a los estudiantes al inicio de curso, que divide cada unidad temática en sesiones de trabajo en el aula. Las fases del trabajo son las siguientes: (a) preparación individual y autónoma de contenidos teóricos; (b) puesta en común y debate de los materiales teóricos; (c) trabajo en grupo: elaboración del guion de una entrevista a una maestra/o; (d) trabajo en grupo autónomo: realización de una entrevista a una maestra/o; (e) trabajo en grupo al aula: elaboración de conclusiones finales.

(a) Preparación individual y autónoma de contenidos teóricos.

Los estudiantes trabajan autónomamente y fuera del aula los materiales proporcionados por el docente para cada uno de los temas. Los materiales a los que pueden acceder los estudiantes, a través de las plataformas virtuales acondicionadas para ese objetivo, son una parte fundamental del aprendizaje. Por tanto, han de estar cuidadosamente elegidos y organizados para poder ser utilizados -si es necesario de manera repetida- teniendo presente que con seguridad será el primer acceso que los estudiantes tengan a la materia impartida. Estos materiales se conforman por breves vídeos documentales, películas o capítulos de series y, por supuesto, textos académicos y de divulgación. Son proporcionados a través del aula virtual teniendo acceso a todos ellos al inicio del curso y estando disponibles hasta la finalización del mismo.

(b) Puesta en común y debate del contenido de los materiales en el aula.

Una vez trabajados individualmente por cada uno de los estudiantes los materiales teóricos proporcionados, se participaba en una relectura colectiva de los mismos. Se trataba de clarificar dudas que hubieran podido surgir en el trabajo autónomo de los materiales pero no sólo. Cambiar el punto de vista desde el cual observamos la realidad dejando de lado aquello que damos por descontado y que forma parte de la construcción social de nuestra cotidianidad 
(Berger y Luckmann, 1988) no es una tarea sencilla, en absoluto. Requiere de un ejercicio metódico de poner en cuestión mucho de lo que hemos aprendido en nuestro proceso de socialización y que conforma nuestra mirada del mundo. Por tanto, consideramos que es necesario que, una vez trabajados los materiales de manera autónoma, el docente ponga énfasis en las cuestiones claves que sustentan estos materiales a partir del cuestionamiento de las ideas y prejuicios que hemos construido alrededor de los mismos. Por ejemplo, en nuestro caso, al referirnos al género como variable estructural, hablábamos de la construcción social de la maternidad o de la importancia de introducir prácticas de coeducación también en las edades más tempranas y de la manera en qué podían responder las familias. Los temas tratados a lo largo del curso e integrados en el temario de la asignatura fueron: el proceso de socialización, la clase social y su influencia en los resultados educativos, el género y la educación, cómo las escuelas atienden el cambio familiar, y la diversidad cultural desde el punto de vista educativo.

(c) Trabajo en grupo: elaboración del guion de una entrevista.

El proceso de aprendizaje iniciado con la preparación autónoma por parte del estudiante de los materiales de la asignatura se consolidaba con la elaboración de un breve guion de preguntas - de tres a cinco preguntas- acerca de cómo influyen o se trabajan en el aula los diferentes temas tratados. Este guion se elaboró en el aula en pequeños grupos (no más de cinco miembros) con el apoyo constante del profesorado. Además, era puesto en común en el conjunto del grupo-aula con el fin de tratar las dudas que pudieran surgir y poder enriquecer el trabajo de cada uno de los grupos con las aportaciones del resto de compañeros/as.

(d) Trabajo en grupo autónomo: realización de una entrevista a una maestra/o.

Una vez elaborado el guion de la entrevista, los estudiantes tenían que buscar a una maestra o maestro de Educación Infantil que estuviera dispuesta a hacer la entrevista, concretar una cita, realizar la entrevista, grabarla y transcribirla. Se trataba de entrevistas breves, no más de diez minutos, a las que accedían a través de sus redes personales. A los maestros/as entrevistados se les proporcionaba un documento firmado por mí como docente de la asignatura en el que se explicaban las razones de la entrevista y cómo iba a ser tratada la información resultante. Además para la realización de la entrevista en unas ciertas condiciones de calidad les proporcionamos unas nociones metodológicas básicas acerca de las condiciones que se tenían que dar en la elaboración de un guion de entrevista pero también en la posición que debían de ocupar como entrevistadores, la gestión del contexto en que se realizaba la entrevista y el tratamiento de la información (Silverman, 1993, 2005; Vallés, 1997). Familiarizarse con los métodos de investigación cualitativos, en definitiva, no en vano un objetivo secundario en esta propuesta también fue que los estudiantes conocieran la existencia de diversas posibilidades de aproximarse a la realidad que les envuelve. Y la Sociología proporciona las herramientas necesarias para acceder a la elaboración y análisis de conocimiento científico lo que contrarresta las asunciones que provienen del sentido común, que estructuran nuestra vida cotidiana y son la base de la solidificación de la desigualdad. 
(e) Trabajo en grupo al aula: elaboración de las conclusiones.

Una vez realizada la entrevista la información que generaba era analizada aplicando los conceptos básicos sobre los que pivotaba el tema en cuestión. Este trabajo de análisis se hizo en dos fases. En primer lugar, en el grupo de trabajo, y más tarde en grupo-aula, es decir, toda la clase puso en común sus ideas y conclusiones extraídas de las entrevistas realizadas. Se consensuaban cuestiones de interés y se debatían cómo en la vida real se podía apreciar, con predisposición, método y conocimiento teórico, aquellas ideas tan abstractas para ellos al dar comienzo el curso. Y cómo el conocimiento científico nos ayuda a entender mejor nuestra cotidianidad.

Hay que señalar que todos los trabajos de los estudiantes (transcripciones, análisis de las entrevistas y conclusiones) eran accesibles a todos los compañeros a través de una plataforma virtual creada a propósito para este fin. Por tanto no sólo podían aprender de su trabajo y del debate que se propiciaba en el aula, sino a partir del trabajo escrito de sus compañeros/as. Se trataba de fortalecer la idea de la importancia de establecer redes de apoyo y de trabajo colaborativo.

En conclusión, toda la propuesta metodológica aplicada tenía como objetivo básico evidenciar la importancia de observar nuestra realidad cotidiana desde la perspectiva del conocimiento científico. Y por tanto, cuestionar todo aquello que en la escuela aceptamos y reforzamos desde el conocimiento de sentido común.

\subsection{RESULTADOS DE LA PROPUESTA}

La evaluación del trabajo realizado por los estudiantes se llevó a cabo en dos niveles. En primer lugar, al final del curso cada grupo entregaba un cuaderno en el que se sintetizaba las conclusiones a las que llegaban en cada uno de los temas a partir del análisis de las entrevistas. Este cuaderno requería de una introducción y de una conclusión final y general de todo el proceso de trabajo. En segundo lugar, la prueba escrita final de evaluación que se basaba en la explicación de los procesos y conceptos teóricos aprendidos a través del estudio autónomo de los materiales proporcionados, así como a través de las entrevistas realizadas y de los debates abiertos durante las sesiones de trabajo que compartimos a lo largo de todo el curso. Es decir que aquello que se valoraba era que los estudiantes hicieran el camino inverso al realizado a lo largo del curso: que su observación de la realidad les ayudara a fundamentar mejor su conocimiento teórico sobre los temas de la materia.

Los resultados previstos al inicio de elaborar esta propuesta se dirigían a mejorar la comprensión por parte del estudiantado de los conceptos básicos de la asignatura en comparación a la conseguido con el uso de metodologías más tradicionales, pero también a lograr una actitud de crítica que cuestionara nuestro conocimiento de sentido común. Además pretendía que los estudiantes se acercaran a las escuelas y que pudieran ver cómo en éstas se perciben y trabajan conceptos claves de la asignatura como el género, la clase social o la diversidad cultural. En este sentido la experiencia ha sido muy positiva. La capacidad para emplear conceptos clave, para expresar las ideas y argumentos de manera adecuada así como su capacidad de preguntarse y repreguntarse por aquello que damos por sentado y con ello cómo la estructura social incide en las escuelas, y esto se ha reflejado en un incremento 
notable de la calidad de los trabajos realizados. Por otro lado, a medida que transcurría el curso se fue apreciando claramente cómo los estudiantes mejoraban de manera significativa sus destrezas y el tiempo que requerían para realizar las tareas asignadas se reducía considerablemente, hasta el punto de necesitar menos sesiones de trabajo por tema. Son conclusiones a las que llegamos como docentes en nuestros encuentros de evaluación del trabajo realizado.

En cuanto a la evaluación por parte de los estudiantes de la propuesta metodológica planteada, se les pasó un pequeño cuestionario anónimo a los estudiantes al inicio y al final de curso con preguntas referidas a cuestiones claves como la estructura social se aplica a la educación con la intención de poder valorar cómo ha evolucionado la comprensión de estos conceptos y procesos. Y en este sentido, en el logro de la imaginación sociológica, estamos muy satisfechos. Pensamos que sí han cambiado la forma de mirar la realidad que los rodea.

En el segundo cuestionario incluimos una pregunta de valoración sobre la asignatura y éstas son las principales conclusiones:

1. Los estudiantes valoran positivamente la colaboración entre sus compañeros/as como una herramienta de aprendizaje que ha procurado una mayor cohesión de grupo. Aunque siempre hay conflictos ha sido muy positivo y provechoso aprender a llegar a acuerdos. Y no se circunscribe al grupo pequeño, también al conjunto de la clase. "Ya que al participar y escuchar de una forma activa te da la oportunidad de pensar y interiorizar los conceptos"

2. Ponen en valor la autonomía y responsabilidad en el proceso de aprendizaje que han tenido en esta propuesta metodológica. "Esta metodología [..] nos posibilita ser más autónomos a la hora de leer las lecturas asignadas a cada tema y ser responsables y conscientes de la importancia de leerlas para poder debatir en grupo"; "Me ha gustado meter en común lo que íbamos haciendo. Era un momento en el que los alumnos éramos escuchados (lo que no siempre ocurre)".

3. También ha sido valorado positivamente el acceso a las escuelas y las conversaciones mantenidas con las y los maestros, lo plantean como asomarse a la realidad que al terminar la titulación deberán ocupar también. Y lo han agradecido mucho:"Nos ha posibilitado visibilizar la realidad que se vive en las escuelas"

Y sobre todo, repiten constantemente, la asignatura - a pesar del desconcierto inicial- les ha ayudado mucho a mirar de manera distinta su cotidianidad, a hacerles ver que detrás de las apariencias hay hilos que desconocían y que hacen funcionar el día a día, y que sobre todo hay desigualdad en cuestiones que entendían como normales. "Me ha despertado las ganas de buscar, seguir leyendo y no dejar los temas que hemos empezado en el olvido".

Sí que ha habido pequeños aspectos negativos todas y todos reiteran el mismo: tener que buscar ellos mismos los materiales: "No me gusta nada tener que buscar el temario por internet o en enciclopedias o demás... creo que es una pérdida de tiempo impresionante y que, al igual que hacen todas las demás profesoras, nos podría facilitar el material por estudiante un poco más". 
En definitiva, la valoración realizada por los estudiantes es claramente satisfactoria. Por supuesto quedan elementos que mejorar, algunos señalados durante el curso por los mismos estudiantes (de nuevo, una muestra del compromiso alcanzado) como la gestión de los tiempos en las sesiones o el calendario de estas mismas sesiones. Pero en definitiva, el objetivo de conseguir por parte de los estudiantes una mayor implicación en la construcción de su aprendizaje y un incremento en la comprensión de conceptos teóricos complejos pero imprescindibles en su futuro día a día como maestros se ha conseguido.

\section{Conclusiones}

Los resultados alcanzados por la propuesta metodológica presentada en este artículo han sido totalmente satisfactorios, tanto para nosotros como docentes como -según las evidencias recogidas- para los estudiantes. A través de metodologías activas de aprendizaje creemos que nos hemos acercado en mayor medida a la meta de la asignatura: aprender cómo la estructura social toma cuerpo en la escuela y al tiempo tratar de enseñar Sociología a quienes no estudian Sociología, lo cual no es una tarea sencilla.

Los objetivos propuestos al inicio del curso fueron ampliamente asumidos. Es decir, el compromiso de los estudiantes en la asignatura y, lo que es más importante, en el proceso de construcción de su aprendizaje fue mayoritario. Fueron muchas las muestras de este compromiso destacando la total implicación de todos los miembros en la mayoría de los grupos en el trabajo marcado, la participación activa en los debates de clase, la asistencia a actividades recomendadas fuera de clase e incluso la participación activa en las mismas o también el interés por mejorar los trabajos realizados con la consulta de los trabajos de los compañeros a través de la plataforma habilitada a esos efectos. Todos estos ejemplos ponen de manifiesto la importancia de fomentar la autonomía de los estudiantes para construir su propia mirada sobre el mundo y que en esta mirada se incluya el conocimiento científico. La independencia que hemos podido observar entre los estudiantes ha sido significativa, aun así consideramos necesario matizar las afirmaciones que frecuentemente se hacen en la defensa de la idoneidad de estas metodologías docentes activas acerca de la transformación del papel del profesor en el aula, representando un rol simplemente de mediador. Consideramos sin embargo que esa transformación es muy limitada dado que los docentes marcamos los límites de la asignatura y sus fines y las maneras de proceder en la clase, de un modo flexible, es cierto, pero las principales decisiones son tomadas por el profesorado que es quien evalúa al final. Se diluye, pero permanece, la relación jerárquica entre estudiantado y profesorado.

Finalmente, se trata de una propuesta que puede ser aplicada a otros grupos de la misma asignatura pero también a otros estudios en los que ocupa la Sociología un lugar en su plan de estudios. La Sociología según Wright Mills (1993) debería tener como principal objetivo conseguir mirar la realidad de otra manera y que eso implicara un cierto compromiso con la realidad que nos envuelve. Como bien apuntan Hironimus-Wendt y Wallace (2009) es importante desarrollar una pedagogía que consiga proporcionar las herramientas suficientes a los estudiantes para poder aplicarla en su vida profesional pero también como ciudadanos, con el fin de conseguir mejorar la vida de sus comunidades. Un elemento importante en una época como la nuestra, donde se considera al individuo el principal responsable de los problemas que le acucian y eso los hace irresolubles puesto que su fuente es claramente 
estructural, generando un profundo malestar (Bauman, 2003, 2007; Béjar, 2007). Esta conciencia social que creemos que se ha conseguido despertar entre una gran parte de los estudiantes lleva incorporada la capacidad de sobrepasar las paredes del aula, lo cual resulta un elemento de mayor riqueza de la propuesta planteada. En conclusión, toda la propuesta metodológica aplicada tenía como objetivo básico evidenciar la importancia de observar nuestra realidad cotidiana desde la perspectiva del conocimiento científico. Y por tanto, cuestionar todo aquello que en la escuela aceptamos y reforzamos desde el conocimiento de sentido común. Y eso se ha conseguido con creces.

\section{Bibliografía}

Angelini, M.Laura (2016) "Integration of the Pedagogical Models "Simulation" and "Flipped Classroom" in Teacher Instruction". SAGE Open, Vol 6, Issue 1. DOI: $10.1177 / 2158244016636430$

Ariño, Antonio; Serra, Inmaculada (2010) "Cultura y socialización” a Garcia Ferrando, Manuel. Pensar nuestra sociedad globalizada. València: Tirant lo Blanch.

Bauman, Zygmunt (2003). "Individualmente, pero juntos. Prefacio" en Beck, Ulrich; BeckGernsheim, Elisabeth. La individualización. Barcelona: Paidós. pp. 19-26.

(2007). La sociedad individualizada. Madrid: Cátedra.

Béjar, Helena (2007) Identitades inciertas: Zygmunt Bauman. Barcelona: Herder.

Berger, Peter L.; Luckmann, Thomas (1988) La construcció social de la realitat: un tractat de sociologia del coneixement. Barcelona: Herder.

Bergmann, Jonathan; Sams, Aaron (2012) Flip your classroom: reach every student in every class every day. Eugene, Oregon: International Society for Technology in Education.

Bain, Ken (2006) El que fan els millors professors d'universitat. València: Universitat de València.

Beltrán, Jose; Hernàndez, Francesc J. (coords.) Sociología de la Educación. Madrid: McGrawHill

Greenfield, Derek (2006) “Understanding Social Structure through Personal Experience: The Creative Use of Status and Role as Explanatory Factors". Teaching Sociology, 34(4):404-11. DOI: $10.1177 / 0092055 X 0603400407$

Fernández Palomares, Francisco (coord.) (2009) Sociología de la Educación. Madrid: Pearson.

Forsey, Martin; Low, Mitchell; Glance, David (2013). "Flipping the sociology classroom: Towards a practice of online pedagogy". Journal of Sociology, vol. 49 (4): 471-485. DOI: $10.1177 / 1440783313504059$

Fuertes Muñoz, Carlos; Cantó Doménech, José (2015). La tècnica de l'itinerari didàctic en la formació inicial del professorat: reconstrucció d'una experiència. @tic. revista d'innovació educativa. ( $\mathrm{n}^{\mathrm{o}}$ 14). DOI: 10.7203/attic. 14.4417

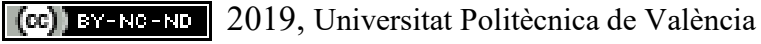


Hattery, Angela J. (2003). "Sleeping in the Box, Thinking Outside the Box: Student Reflections on Innovative Pedagogical Tools for Teaching about and Promoting a Greater Understanding of Social Class Inequality among Undergraduates". Teaching Sociology, 31(4):412-27.

Hironimus-Wendt, Robert J.; Wallace, Lora Ebert. (2009). "The Sociological Imagination and Social Responsibility. Teaching Sociology 37(1):76-88. DOI: 10.1177/0092055X0903700107

Huisman, Kimberly (2010) "Developing a Sociological Imagination by Doing Sociology: A Methods-based Service-learning Course on Women and Immigration. Teaching Sociology 38(2) 106-118. DOI: 10.1177/0092055X10364013

Hsiung, Ping-Chun (2008) "Teaching Reflexivity in Qualitative Interviewing". Teaching Sociology, Vol. 36. 211-226. DOI: 10.1177/0092055X0803600302

Lage, Maureen J; Platt, Glenn J; Treglia, Michael (2000) "Inverting the classroom: A gateway to creating an inclusive learning environment". Journal of Economic Education, 31, 1, 30-43. DOI: $10.1080 / 00220480009596759$

Reidsema, Carl; Kavanagh, Lydia; Hadgraft, Roger; Smith, Neville (2017) The Flipped Classroom: Practice and Practices in Higher Education. Singapore: Springer Singapore.

Silverman, David (1993) Interpreting qualitative data: methods for analysing talk, text and interaction. London: Sage.

(2005) Doing qualitative research: a practical handbook. London: Sage.

Vallés, Miguel (1997) Técnicas cualitativas de investigación social: reflexión metodológica y práctica profesional. Madrid: Síntesis.

Wright Mills, C. (1993) La imaginación sociológica. 14a reimpr. México: Fondo de Cultura Económica. 